\title{
Feasibility of measuring renal blood flow by phase-contrast magnetic resonance imaging in patients with autosomal dominant polycystic kidney disease
}

\author{
E. M. Spithoven ${ }^{1}$ - E. Meijer ${ }^{1}$ - C. Borns ${ }^{2}$ - W. E. Boertien ${ }^{1}$ - C. A. J. M. Gaillard ${ }^{1}$. \\ P. Kappert ${ }^{2}$ - M. J. W. Greuter ${ }^{2}$ - E. van der Jagt ${ }^{2}$ • P. Vart ${ }^{3}$ • P. E. de Jong ${ }^{1}$ • \\ R. T. Gansevoort ${ }^{1}$
}

Received: 14 September 2014 / Revised: 2 June 2015 / Accepted: 3 June 2015 / Published online: 11 July 2015

(C) The Author(s) 2015. This article is published with open access at Springerlink.com

\begin{abstract}
Objectives Renal blood flow (RBF) has been shown to predict disease progression in autosomal dominant polycystic kidney disease (ADPKD). We investigated the feasibility and accuracy of phase-contrast RBF by MRI ( $\left.\mathrm{RBF}_{\mathrm{MRI}}\right)$ in ADPKD patients with a wide range of estimated glomerular filtration rate (eGFR) values.

Methods First, we validated $\mathrm{RBF}_{\mathrm{MRI}}$ measurement using phantoms simulating renal artery hemodynamics. Thereafter, we investigated in a test-set of 21 patients intra- and interobserver coefficient of variation of $\mathrm{RBF}_{\mathrm{MRI}}$. After validation, we measured $\mathrm{RBF}_{\mathrm{MRI}}$ in a cohort of 91 patients and compared the variability explained by characteristics indicative for disease severity for $\mathrm{RBF}_{\mathrm{MRI}}$ and $\mathrm{RBF}$ measured by continuous hippuran infusion.

Results The correlation in flow measurement using phantoms by phase-contrast MRI was high and fluid collection was high $(\mathrm{CCC}=0.969)$. Technical problems that precluded $\mathrm{RBF}_{\mathrm{MRI}}$ measurement occurred predominantly in patients with a lower
\end{abstract}

Electronic supplementary material The online version of this article (doi:10.1007/s00330-015-3877-y) contains supplementary material, which is available to authorized users.

R. T. Gansevoort

R.T.Gansevoort@umcg.nl

1 Department of Nephrology, Community and Occupational Medicine, University Medical Center Groningen, University of Groningen, PO Box 30.001, 9700 RB Groningen, The Netherlands

2 Department of Radiology, Community and Occupational Medicine, University Medical Center Groningen, University of Groningen, Groningen, The Netherlands

3 Department of Health Sciences, Community and Occupational Medicine, University Medical Center Groningen, University of Groningen, Groningen, The Netherlands
eGFR (34\% vs. 16\%). In subjects with higher eGFRs, variability in RBF explained by disease characteristics was similar for $\mathrm{RBF}_{\mathrm{MRI}}$ compared to $\mathrm{RBF}_{\mathrm{Hip}}$, whereas in subjects with lower eGFRs, this was significantly less for $\mathrm{RBF}_{\mathrm{MRI}}$.

Conclusions Our study shows that RBF can be measured accurately in ADPKD patients by phase-contrast, but this technique may be less feasible in subjects with a lower eGFR.

Key points

- Renal blood flow (RBF) can be accurately measured by phase-contrast MRI in ADPKD patients.

- RBF measured by phase-contrast is associated with ADPKD disease severity.

- RBF measurement by phase-contrast MRI may be less feasible in patients with an impaired eGFR.

Keywords Renal blood flow measurement · Phase-contrast MRI · Hippuran · ADPKD · Phantom

\section{Introduction}

Most autosomal dominant polycystic kidney disease (ADPKD) subjects show progressive kidney function decline and develop end-stage renal disease (ESRD) between their 4th to 7 th decade of life [1]. With renoprotective treatments emerging, such as vasopressin V2-receptor antagonists and somatostatin analogues [2, 3], it will be important to assess disease severity. Especially those patients with a high likelihood of disease progression towards ESRD will need treatment. Assessing disease severity and prognosis in ADPKD is not without difficulty. Glomerular filtration rate (GFR) may not be the best measure in this respect, because GFR remains within normal limits for an extended period of time [4]. Therefore, total kidney volume (TKV) assessed by MRI has been 
investigated as an alternative [5]. TKV measurement is, however, laborious and the association between TKV and disease severity is limited at an individual patient level. Renal blood flow (RBF) has been proposed as a third marker to assess disease severity [6]. A decrease in RBF flow paralleled TKV growth in the observational CRISP cohort study [6] and is thought to precede decline in GFR [7].

However, the value of RBF has been investigated in a single study, using gadolinium-contrast to obtain a reference image of the renal artery to position the phase-contrast perpendicular to the vessel contour and included only ADPKD subjects with a creatinine clearance $>70 \mathrm{ml} / \mathrm{min}[6,7]$. Confirmation, especially in subjects with lower kidney function, is therefore needed. Moreover, a method without gadolinium administration would be preferable to avoid the risks of developing gadolinium-associated contrast-induced nephropathy [8] and nephrogenic systemic fibrosis [9, 10]. Besides MRI, RBF can also be measured by infusion of para-aminohippuric acid $\left(\mathrm{RBF}_{\mathrm{Hip}}\right)[11,12]$.

Given these considerations, the aim of the present study was to investigate whether measurement of RBF by MRI without using gadolinium contrast $\left(\mathrm{RBF}_{\mathrm{MRI}}\right)$ could be performed accurately and precisely in a group of ADPKD patients with a wide range in kidney function. First, we validated $\mathrm{RBF}_{\mathrm{MRI}}$ measurement using phantoms simulating renal artery hemodynamics. Thereafter, we investigated in a test-set of patients the intra- and inter-observer variability. After validation, we compared $\mathrm{RBF}_{\mathrm{MRI}}$ with $\mathrm{RBF}_{\mathrm{Hip}}$, and investigated associations of $\mathrm{RBF}_{\mathrm{MRI}}$ and $\mathrm{RBF}_{\mathrm{Hip}}$ with characteristics indicative for disease severity in ADPKD subgroups.

\section{Methods and Materials}

\section{Patient population}

For this study, all consecutive patients with ADPKD visiting our out-patient clinic from January 2007 until September 2012 were asked to participate. The diagnosis of APDKD was made based upon the revised Ravine criteria [13]. Subjects were considered ineligible if they received renal replacement therapy, had undergone renal surgery, were unable to undergo MRI, and had other systemic diseases or treatments potentially affecting kidney function. One hundred forty six subjects met these criteria, and were invited for an extensive, one-day screening. This screening included, among others, an MRI and assessment of effective renal plasma flow (ERPF), which were used to measure $\mathrm{RBF}_{\mathrm{MRI}}$ and $\mathrm{RBF}_{\mathrm{Hip}}$. Thirteen patients refused to participate and in thirteen patients, $\mathrm{RBF}_{\mathrm{MRI}}$ was not measured due to logistical reasons, leaving 120 patients for the present analyses. This study was conducted in accordance with the guidelines of the Helsinki Declaration. All participants gave written informed consent.

\section{Measurement and calculations}

All participants collected at home a 24-hour urine sample the day preceding the screening at our facility, in which albuminuria was measured. During the visit, blood pressure was measured (Dinamap) and fasting blood samples were drawn for determination of osmolality, copeptin and creatinine. GFR was estimated using the CKD-EPI (Chronic Kidney Disease Epidemiology Collaboration) equation [14, 15]. MRI and measurement of renal hemodynamic parameters were performed per protocol on the same day.

\section{Renal blood flow measured by MRI}

Imaging was performed on a 1.5-Tesla Magnetom Avento (Siemens, Erlangen, Germany). Two coils were placed on the anterior and posterior abdominal walls directly over the kidneys. Two fast, respiratory-triggered, coronal and transversal T2-weighted image sets were acquired to determine the kidney volumes [16]. To localize the renal arteries, coronal and transversal data sets were scanned during the breath hold using a T2-weighted TrueFISP sequence. RBF was assessed with phase-contrast MRI (PC-MRI), using a two-dimensional fast gradient echo phase-contrast sequence with retrospective cardiac gating (30 cardiac phases) and flow compensation [17]. Acquisition parameters TR/TE/FA were $43.6 \mathrm{~ms} / 4 \mathrm{~ms} /$ $30^{\circ}$. A single slice was positioned perpendicular to the renal artery. Slice thickness was $5 \mathrm{~mm}$, FOV was $32 \mathrm{~cm}$, and the number of phase-encoding steps was 260-384, depending on patient size. Acquisition time was $150-160$ seconds. The encoding velocity (VENC) was $100 \mathrm{~cm} / \mathrm{s}$ and flow acquisition was obtained in the slice direction. Supplementary Figure 1 shows a representative sample of data recorded during the cardiac cycle and shows a realistic renal arterial waveform. $\mathrm{RBF}_{\mathrm{MRI}}$ measurement lasted between 150 and 160 seconds. Flow analysis was performed using ARGUS flow software (Siemens, Erlangen, Germany) for phase-contrast imaging. Images were magnified to 5.0 and renal artery contours were drawn manually on anatomical images to define the region of interest for the corresponding phase-contrast images at all phases of the cardiac cycle. The drawing of these contours required 5-10 minutes per kidney. Flow rates of all renal arteries (left, right and any additional arteries) were added to calculate total $\mathrm{RBF}_{\mathrm{MRI}}$. Flow measurements were judged technically unsatisfactory when they met one or more of the following criteria: indistinct vessel contour (blurred as a result of motion), artefacts in the vessel area, ghosting (movement artefacts), or non-physiologic, heterogeneous measurement or weak signal [18]. TKV was measured on T2-weighted coronal images using Analyze Direct 8.0 (Analyze Direct, Inc., Overland Park, KS, USA) and divided by height. Intra- and interobserver coefficients of variation for TKV measurement were 1.8 and $2.3 \%$, respectively. 


\section{Validation of flow measured by MRI in a phantom}

Two flexible silicon phantoms, each representing the aorta and two renal arteries perpendicular to the aorta (Elastrat, Geneva, Switzerland) (Fig. 1) were used to assess accuracy of the PCMRI measurements. The four renal arteries in these two phantoms had diameters of 5, 6, 7 and $8 \mathrm{~mm}$, representing the normal range of renal artery diameters. The phantoms were suspended in a container filled with water. An MRIcompatible pump (Cardioflow 1000MR, Shelly Medical Imaging Technologies, London, Canada) was positioned proximally; one renal artery was connected to a container, the other renal artery and distal aorta were connected to a reservoir (Fig. 1). A mix of 40-\% glycerol and $60-\%$ purified water was used as flow medium to mimic blood viscosity $(\sim 3.5$ $\mathrm{mPa} / \mathrm{s})$ [19]. A synchronized trigger pulse signal was generated by the MRI to simulate electrocardiographic (ECG) gating at a heart rate of 60 beats per minute. The flow in the phantom was determined simultaneously by PC-MRI according to the protocol described above and by fluid collection (volume pumped through the renal artery was collected in a container). These flow collections were considered the gold-standard measure to evaluate the accuracy of the MR-calculated flow. Importantly, the system was positioned in the horizontal plane and the container was placed floating in the reservoir to prevent any steady flow bias due to the principle of communicating vessels (Fig. 1). During our phantom experiment, the pump was set at different flow rates (800, 1000, 1200 and $1400 \mathrm{ml} / \mathrm{min}$ ) and acted as "cardiac output". In this phantom, "cardiac output" is divided over two renal arteries and the aorta. Thus, the blood flow rates in the phantom renal arteries were dependent on the pump rates ("cardiac output") and the diameters of the phantom renal arteries and aorta. Flow was

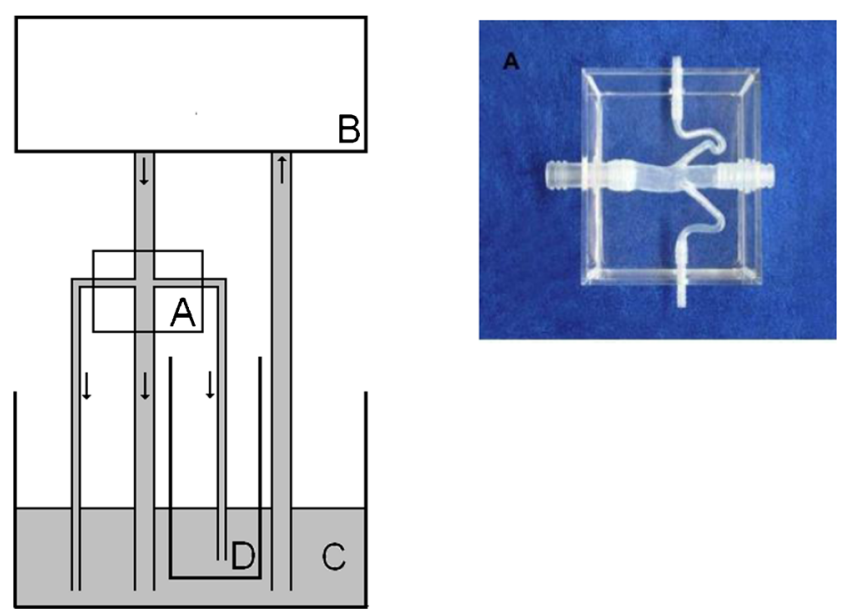

Fig. 1 Schematic representation of the phantom used to study accuracy and reliability of flow measured by MRI when compared to fluid collection: $A$ silicon phantom of the aorta and two renal arteries; $B$ pulsatile pump; $C$ reservoir; and $D$ container in which fluid output was collected for flow measurement measured in one renal artery at a time, but several times to assess reproducibility.

\section{Variability of $\mathrm{RBF}_{\mathrm{MRI}}$ measurement}

$\mathrm{RBF}_{\mathrm{MRI}}$ measurements were performed by two research physicians (ES and EM) whom were trained to perform $\mathrm{RBF}_{\mathrm{MRI}}$ measurements. During their training period, they measured 60 $\mathrm{RBF}_{\mathrm{MRI}}$ values under supervision of an experienced MRI technician (PK). Subsequently, they measured $\mathrm{RBF}_{\mathrm{MRI}}$ on 2 occasions, blinded to previous results, in 21 patients (i.e., 42 kidneys) to determine intra- and inter-observer variability.

\section{RBF measured by ${ }^{131}$ I-hippuran}

Measurement of renal hemodynamic parameters was performed using a constant infusion method with ${ }^{131}$ I-hippuran to measure ERPF $[11,20]$. During these assessments, patients were not water-loaded, but allowed to drink ad libitum. At $08.00 \mathrm{am}$, a priming solution of $0.04 \mathrm{~mL} / \mathrm{kg}$ body weight was administered, followed by a constant infusion of the tracer at a rate depending on eGFR for 5.5 hours. After a stabilisation period of 1.5 hours, two 2-hour clearance periods followed, in which ERPF was measured as $\left(I^{*} \mathrm{~V}\right) / \mathrm{P}$, where $\mathrm{I}^{*} \mathrm{~V}$ represents the infusion of the tracer and $\mathrm{P}$ represents the tracer concentration in plasma. Intra-subject day-to-day variability using this method is $5 \%$ [11]. $\mathrm{RBF}_{\text {Hip }}$ was calculated using the formula $\mathrm{ERPF} /(1$-hematocrit).

\section{Statistical analysis}

Lin's concordance correlation coefficient (CCC) and BlandAltman analyses were used to assess agreement between the gold standard fluid collection and the flow measured by MRI in the phantom. Reproducibility was assessed by CCC, BlandAltman analyses and intra- and inter-observer coefficient of variation $(\mathrm{CV})$.

Subsequently, $\mathrm{RBF}_{\mathrm{MRI}}$ and $\mathrm{RBF}_{\mathrm{Hip}}$ were measured in an ADPKD patient cohort of which baseline characteristics were calculated for the overall population and for participants with an eGFR $>70$ and $\leq 70 \mathrm{ml} / \mathrm{min}^{*} 1.73 \mathrm{~m}^{2}$, separately. Normality was assessed by a Q-Q-plot. Normal distributed variables were expressed as the mean \pm standard deviation (SD), whereas non-normal distributed variables were expressed as the median (interquartile range). Difference in baseline characteristics between two eGFR subgroups was tested using a Chisquare test for categorical data, and a Student's t-test when normally distributed or a Mann-Whitney test when not normally distributed.

Disease characteristics were correlated to $\mathrm{RBF}_{\text {Hip }}$ and to $\mathrm{RBF}_{\mathrm{MRI}}$ in the overall cohort and in subcohorts stratified for eGFR $>70$ and $\leq 70 \mathrm{ml} / \mathrm{min}^{*} 1.73 \mathrm{~m}^{2}$. To investigate the best fit for $\mathrm{RBF}_{\mathrm{Hip}}$ and $\mathrm{RBF}_{\mathrm{MRI}}$, we performed a stepwise backwards 
regression analysis. Clinically important disease characteristics were entered and deleted stepwise from the model in order of weakest association until a model with the best fit (i.e., adjusted $\mathrm{R}^{2}$ ) was obtained. Model discrimination was determined by bootstrapping (2000 times) to calculate the difference in adjusted $\mathrm{R}^{2}$ of two multivariate linear regression models [21].

All analyses were performed with SPSS version 20.0 (SPSS Inc., Chicago, IL, USA). A two-sided $p$-value less than 0.05 was considered to indicate statistical significance.

\section{Results}

\section{Validation of flow measured by MRI}

Figure 2 shows a scatter-plot comparing pulsatile flow measured by fluid collection and flow measured by PC-MRI, combined for all flow rates and vessel diameters. The 5-mm diameter renal artery images were judged technically unsatisfactory, because of artefacts in the vessel area and measurement heterogeneity. An excellent agreement between flow measured by the gold standard of fluid collection and by PC-MRI was observed. The CCC for 6 to $8 \mathrm{~mm}$ was 0.969 $(p<0.001)$, indicating high agreement. Bland-Altman analysis shows that flow measured by MRI on average underestimated flow measured by fluid collection by $2.9 \%$ (Fig. 2). A significant correlation was noted between the mean (flow measured by fluid collection and by MRI) and the absolute difference between these two measures $(\mathrm{r}=-0.47, p=$ $0.004)$.

\section{Reproducibility of $\mathbf{R B F}_{\mathrm{MRI}}$ measurement in a test-set of ADPKD patients}

Figure 3 shows the agreement between blood flow measured by observers ES and EM in 21 ADPKD patients. The average intra-observer CV was $2.3 \%$ (ES $2.0 \%$ and EM $2.6 \%$ ). The estimated reliability, i.e., intra-class correlation coefficient, for observers ES and EM were 0.997 and 0.995 , respectively (both $p<0.001$ ). The inter-observer CV was $3.5 \%$ with a reliability coefficient of $0.991(p<0.001)$. In patients with an eGFR $>70 \mathrm{ml} / \mathrm{min}^{*} 1.73 \mathrm{~m}^{2}$, the intra- and inter-observer CVs were 1.5 and $3.5 \%$, respectively, whereas in patients with an eGFR $\leq 70 \mathrm{ml} / \mathrm{min}^{*} 1.73 \mathrm{~m}^{2}$, these values were 3.2 and $3.5 \%$, respectively.

\section{$\mathrm{RBF}_{\text {MRI }}$ measurement in an ADPKD patient cohort}

Out of the 120 patients, 29 (24\%) had RBF measurements that were judged technically unsatisfactory, and these patients were excluded for further analysis, leaving 91 ADPKD patients for analyses (in patients with an eGFR $\leq 70$ and $>70$
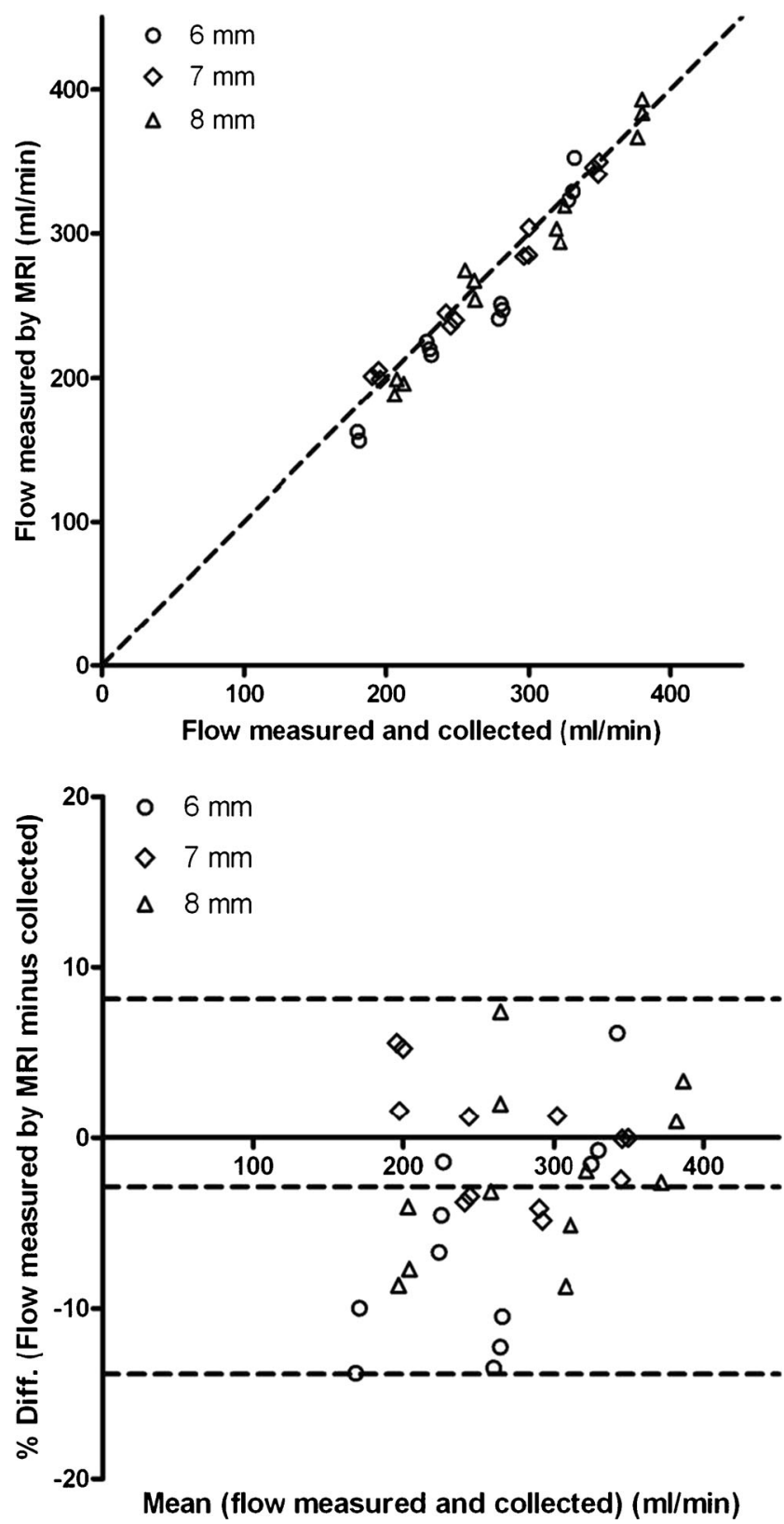

Fig. 2 Results of the phantom experiment. The upper panel shows a scatter plot with flow assessed by fluid collection vs. measured by MRI. The dotted line shows the line of identity. The lower panel shows a corresponding Bland-Altman plot. The CCC was 0.977, 0.989 and 0.933 for the 6,7 and $8 \mathrm{~mm}$ phantoms, respectively $(p<0.001$ for all)

$\mathrm{ml} / \mathrm{min}^{*} 1.73 \mathrm{~m}^{2}$, this held for $18(34 \%)$ and 11 (16\%) patients, respectively). The excluded patients had a significantly lower eGFR and ERPF compared to the patients that could be studied [eGFR 79.2 vs. $58.1 \mathrm{ml} / \mathrm{min}^{*} 1.73 \mathrm{~m}^{2}(p=0.001)$ ], and ERPF 263.5 vs. $201.7 \mathrm{ml} / \mathrm{min}^{*} 1.73 \mathrm{~m}^{2}$ ( $\left.p=0.004\right)$. No significant difference in TKV was observed between the groups (969 vs. $1526 \mathrm{~mL} / \mathrm{m}, p=0.28$ ). The included patients were relatively young with a mean age of $39.8 \pm 11.4$ years and their eGFR ranged from 15 to $136 \mathrm{ml} / \mathrm{min}^{*} 1.73 \mathrm{~m}^{2}$. Nine patients $(9.9 \%)$ had multiple renal arteries (one bilateral, seven 

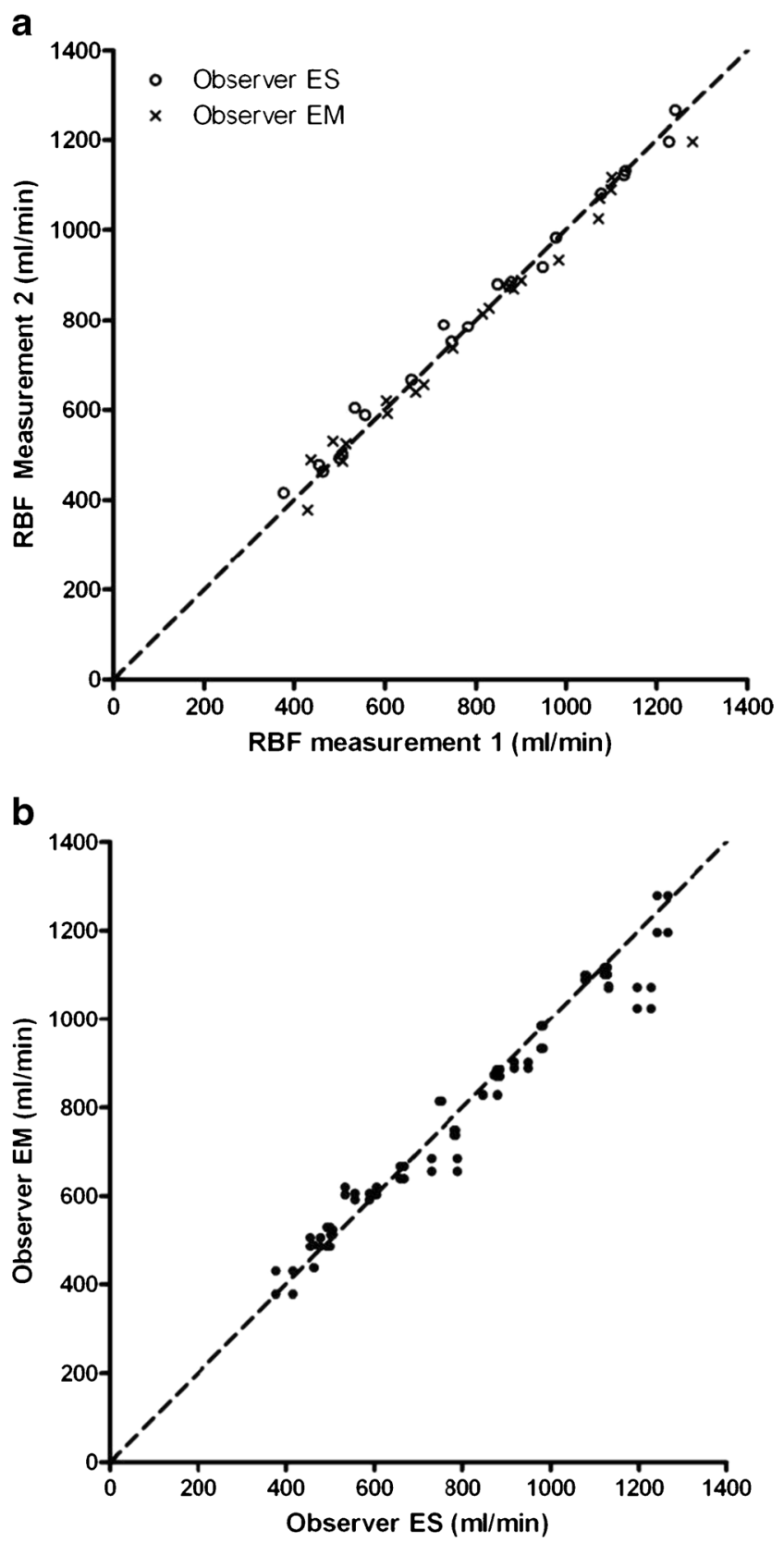

Fig. 3 Intra-observer and inter-observer agreement of renal blood flow (RBF) measurement ( $\mathrm{ml} / \mathrm{min})$. $A$ The intra-class correlation coefficient for observer A was 0.997 and 0.995 for observer B ( $p<0.001$ for both; $\mathrm{n}=21$ ADPKD patients); the intra-observer coefficient of variation was $2.0 \%$ and $2.6 \%$, respectively. $B$ The inter-observer coefficient of variation was $3.5 \%$ with a reliability coefficient of $0.991(p<0.001)$

unilateral and one unilateral with two additional renal arteries). $\mathrm{RBF}_{\mathrm{MRI}}$ ranged from 244 to $1547 \mathrm{ml} / \mathrm{min}^{*} 1.73 \mathrm{~m}^{2}$. Right artery $\mathrm{RBF}_{\mathrm{MRI}}$ was significantly higher than left artery $\mathrm{RBF}_{\text {MRI }}$ (456 vs. $\left.358 \mathrm{ml} / \mathrm{min} * 1.73 \mathrm{~m}^{2}, p<0.001\right)$. The smallest renal artery diameter was 3.9 and the largest was $11.4 \mathrm{~mm}$. The additional renal arteries had smaller diameters, with a mean of $6 \mathrm{~mm}$ compared to $7 \mathrm{~mm}$ of "single" renal arteries. As expected, RBF was significantly lower in patients with lower eGFR whereas age, TKV and use of antihypertensives were significantly higher in this subgroup (Table 1). Figure 4 shows examples of PC-MRI flows that were judged technically satisfactory and unsatisfactory.

\section{Associations of $\mathrm{RBF}_{\mathrm{MRI}}$ and $\mathrm{RBF}_{\mathrm{Hip}}$ with disease characteristics.}

$\mathrm{RBF}_{\text {MRI }}$ correlated significantly with $\mathrm{RBF}_{\mathrm{Hip}}(\mathrm{r}=0.81, p<$ 0.001 ), although $\mathrm{RBF}_{\mathrm{MRI}}$ was higher than $\mathrm{RBF}_{\text {Hip }}$ over the entire range of $\mathrm{RBF}_{\mathrm{MRI}}$. In univariate regression analyses, both $\mathrm{RBF}_{\mathrm{MRI}}$ as well as $\mathrm{RBF}_{\mathrm{Hip}}$ were positively correlated with eGFR and urinary sodium excretion, and negatively with age and TKV (Table 2). A stepwise backward multiple regression model showed that $\mathrm{RBF}_{\mathrm{Hip}}$ and $\mathrm{RBF}_{\mathrm{MRI}}$ were significantly and especially associated with eGFR (both $<0.001$ ). In the whole group, the variability explained by the model for $\mathrm{RBF}_{\mathrm{MRI}}$ was significantly lower than for $\mathrm{RBF}_{\text {Hip }}$ (adjusted $\mathrm{R}^{2}=0.61$ and 0.77 , respectively; $p<0.001$ ). In patients with an eGFR $>70 \mathrm{ml} / \mathrm{min}^{*} 1.73 \mathrm{~m}^{2}$, the adjusted $\mathrm{R}^{2}$ was not significantly different for $\mathrm{RBF}_{\mathrm{MRI}}$ than $\mathrm{RBF}_{\text {Hip }}(0.36$ and 0.51 , respectively; $p=0.19$ ), whereas in patients with an eGFR $\leq 70 \mathrm{ml} / \mathrm{min}^{*} 1.73 \mathrm{~m}^{2}$, disease characteristics explained a smaller fraction of variability in $\mathrm{RBF}_{\mathrm{MRI}}$ compared to $\mathrm{RBF}_{\mathrm{Hip}}$ ( 0.17 and 0.72 , respectively; $p<0.001$; Table 3 ).

In addition, we performed a sensitivity analysis in which we excluded patients with additional renal arteries in order to investigate whether multiple renal arteries caused methodological problems. Multiple renal arteries were found only in patients with eGFR $>70 \mathrm{ml} / \mathrm{min}^{*} 1.73 \mathrm{~m}^{2}$. We found similar variability in measurement of $\mathrm{RBF}_{\mathrm{MRI}}$ and $\mathrm{MRI}_{\mathrm{Hip}}$ as in the whole group (adjusted $\mathrm{R}^{2}=0.63$ and 0.77 , respectively), and the variability explained by the model increased slightly for $\mathrm{RBF}_{\mathrm{MRI}}$ (adjusted $\mathrm{R}^{2}=0.42$ ) and remained similar for $\mathrm{RBF}_{\mathrm{Hip}}$ (adjusted $\mathrm{R}^{2}=0.53$ ).

\section{Discussion}

In this study, we investigated whether measurement of RBF by MRI without using gadolinium contrast $\left(\mathrm{RBF}_{\mathrm{MRI}}\right)$ could be performed accurately and precisely. First, we validated $\mathrm{RBF}_{\mathrm{MRI}}$ measurement using phantoms simulating renal artery hemodynamics. Thereafter, we investigated in a test-set of patients the reproducibility of $\mathrm{RBF}_{\mathrm{MRI}}$. After validation, we measured $\mathrm{RBF}_{\mathrm{MRI}}$ in a cohort of ADPKD patients and compared $\mathrm{RBF}_{\mathrm{MRI}}$ and $\mathrm{RBF}$ measured by hippuran infusion with characteristics indicative for disease severity.

In our phantom experiments, mimicking the clinical renal hemodynamic profile, $\mathrm{RBF}_{\mathrm{MRI}}$ showed perfect agreement to the gold-standard for flow measurement, i.e., fluid collection. We observed no differences in accuracy in $\mathrm{RBF}_{\mathrm{MRI}}$ between the "renal arteries" varying in diameter or between flow 
Table 1 Baseline characteristics of the 91 ADPKD patients included in the present study

\begin{tabular}{|c|c|c|c|c|}
\hline & \multirow[t]{2}{*}{ All } & \multicolumn{3}{|c|}{ Stratified according to eGFR $\left(\mathrm{ml} / \mathrm{min}^{*} 1.73 \mathrm{~m}^{2}\right)$} \\
\hline & & $>70$ & $\leq 70$ & $p$-value \\
\hline Number of participants & 91 & 57 & 34 & \\
\hline Age (y) & $39.8 \pm 11.4$ & $34.9 \pm 8.5$ & $48.0 \pm 11.2$ & $<0.001$ \\
\hline Male $(\%)$ & $58.2 \%$ & $54.4 \%$ & $64.7 \%$ & 0.3 \\
\hline Diastolic BP (mm Hg) & $79.9 \pm 9.6$ & $79.9 \pm 9.7$ & $79.9 \pm 9.5$ & 0.9 \\
\hline Systolic BP (mm Hg) & $129.2 \pm 11.9$ & $129.4 \pm 12.0$ & $128.9 \pm 12.0$ & 0.8 \\
\hline Antihypertensive medication (\%) & $79.1 \%$ & $70.2 \%$ & $94.1 \%$ & 0.007 \\
\hline Plasma Osmolality (mOsm/kg) & $288 \pm 6.7$ & $286.5 \pm 6.5$ & $290.8 \pm 6.2$ & 0.004 \\
\hline Serum Copeptin (pmol/l) & $6.5(3.0-14.7)$ & $4.4(2.7-8.8)$ & $13.2(8.0-18.1)$ & $<0.001$ \\
\hline eGFR (ml/min*1.73m²) & $79.2 \pm 28.8$ & $98.1 \pm 15.3$ & $47.5 \pm 14.7$ & $<0.001$ \\
\hline Renal blood flow (MRI) (ml/min*1.73m²) & $814 \pm 302$ & $973.6 \pm 243$ & $545 \pm 174$ & $<0.001$ \\
\hline Renal blood flow (hippuran) (ml/min*1.73m²) & $440 \pm 157$ & $533 \pm 109$ & $285 \pm 86.7$ & $<0.001$ \\
\hline Total kidney volume $(\mathrm{mL} / \mathrm{m})$ & $852(251-1272)$ & $729(483-1083)$ & $1167(748-1434)$ & 0.003 \\
\hline $24 \mathrm{~h}$ urinary volume $(\mathrm{mL})$ & $2255 \pm 767$ & $2173 \pm 842$ & $2392 \pm 613$ & 0.2 \\
\hline 24h urinary sodium excretion (mmol) & $173 \pm 71$ & $184 \pm 74$ & $157 \pm 65$ & 0.1 \\
\hline $24 \mathrm{~h}$ urinary albumin excretion (mg) & $36.3(14.2-117.8)$ & $36.3(14.8-88.0)$ & $42.4(13.3-178.4)$ & 0.4 \\
\hline
\end{tabular}

Note: Unless otherwise indicated, values for categorical variables are given as percentages; values for continuous variables are given as the mean \pm standard deviation (if parametric) or median [interquartile range] if nonparametric. $P$-values indicate differences between $>70 \mathrm{ml} / \mathrm{min}^{*} 1.73 \mathrm{~m}^{2}$ and $\leq 70$ $\mathrm{ml} / \mathrm{min}^{*} 1.73 \mathrm{~m}^{2}$

$P$-values are calculated by t-tests when normally distributed and by Mann-Whitney U tests when non-normally distributed. Abbreviations: ADPKD, autosomal dominant polycystic kidney disease; BP, blood pressure; eGFR, estimated glomerular filtration rate

velocities, although it should be noted that measurement of $\mathrm{RBF}_{\mathrm{MRI}}$ was not feasible in the "artery" with the smallest diameter $(5 \mathrm{~mm})$. Our findings are in line with the values obtained by Dambreville et al [19], who showed that steady and pulsatile flow can be measured reliably in polyvinyl alcohol tubes with different diameters. These authors also experienced difficulties in measuring MRI flow in tubes with small diameters. In our test set of 21 patients, reproducibility was high and comparable with values obtained in the literature with contrast-measured RBF $[6,19]$. These data indicate that $\mathrm{RBF}_{\mathrm{MRI}}$ can be measured reliably in vitro and in vivo. In patients without any additional renal arteries, we found similar variability in measurement of $\mathrm{RBF}_{\mathrm{MRI}}$ and $\mathrm{MRI}_{\mathrm{Hip}}$ compared to all patients. These data indicate that the presence of additional renal arteries also does not influence the reliability of RBF measurement.

We used MRI to measure $\mathrm{RBF}_{\mathrm{MRI}}$ in a cohort of ADPKD patients. Our technical success rate was $76 \%$, which is comparable with previous literature on this method [18]. We compared $\mathrm{RBF}_{\mathrm{MRI}}$ with $\mathrm{RBF}_{\mathrm{Hip}}$ in these patients. It should be noted that $\mathrm{RBF}_{\mathrm{Hip}}$ is another indirect measurement of RBF. $\mathrm{RBF}_{\text {Hip }}$ was lower compared to $\mathrm{RBF}_{\mathrm{MRI}}$ in patients with relatively preserved as well as impaired kidney function. It is known that $\mathrm{RBF}_{\text {Hip }}$ underestimates RBF, because the extraction of hippuran by tubular cells is not $100 \%$ and dependent on kidney function [12]. In two previous studies, it was shown that $\mathrm{RBF}_{\mathrm{MRI}}$ correlated well with $\mathrm{RBF}$ determined by clearance of para-amino-hippurate $(r=0.94$ and $r=0.93$, respectively) $[22,23]$. These studies used healthy subjects. In the present study we applied hippuran instead of paraamino-hippurate [12]. We showed that $\mathrm{RBF}_{\mathrm{MRI}}$ correlated well with $\mathrm{RBF}_{\text {Hip }}(\mathrm{r}=0.90)$ and that $\mathrm{RBF}_{\mathrm{MRI}}$ was associated with anatomical, hemodynamic and laboratory parameters indicating disease severity. This corroborates the findings by the CRISP consortium that found the same associations and additionally described that $\mathrm{RBF}_{\mathrm{MRI}}$ predicted kidney function decline [6]. Measurement of RBF can, therefore, be important to assess disease severity, to predict prognosis and potentially also act as a surrogate endpoint to assess the efficacy of renoprotective interventions early in the disease. RBF could also be used in combination with GFR to assess the filtration fraction [24].

Although on average we showed that RBF could be measured reliably by MRI without using contrast, there are a number of issues rendering such measurement less feasible in ADPKD patients with impaired kidney function. First, $\mathrm{RBF}_{\mathrm{MRI}}$ measurement in the smallest phantom artery (5 $\mathrm{mm}$ ), which is a surrogate for more severe ADPKD, was not possible. Second, in the phantom experiment, we found a significant correlation between mean flow and difference in flow measured by MRI and fluid collection, with a larger difference in the lower flow range. This indicates that systematic bias increases at the lower flow range. Third, variability was higher in patients with a low eGFR than in patients with a 
Fig. 4 Phase-contrast magnetic resonance angiogram without gadolinium of the renal arteries in ADPKD. a The phase-contrast angiogram was performed perpendicular to the renal artery on the coronal image b Crosssectional image through the renal artery that was judged technically satisfactory and $\mathbf{c}$ unsatisfactory to measure renal blood flow, because of indistinct vessel jcontour (blurred as a result of motion)

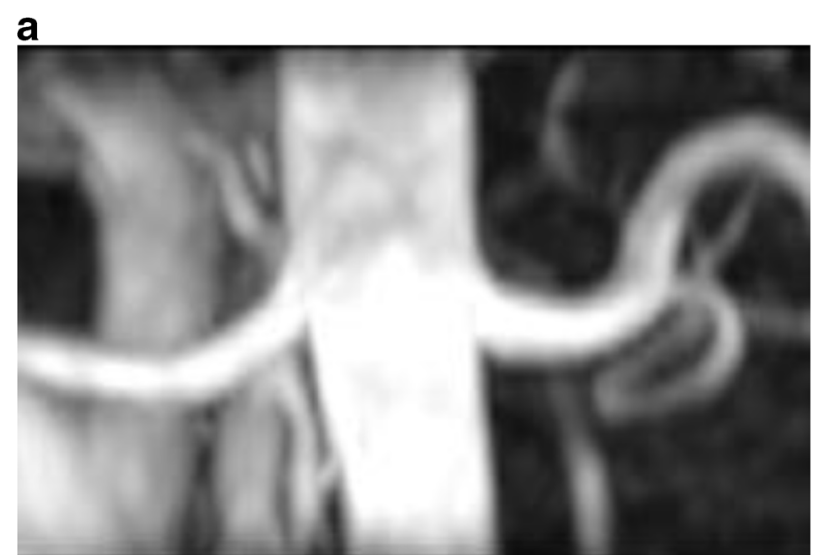

b

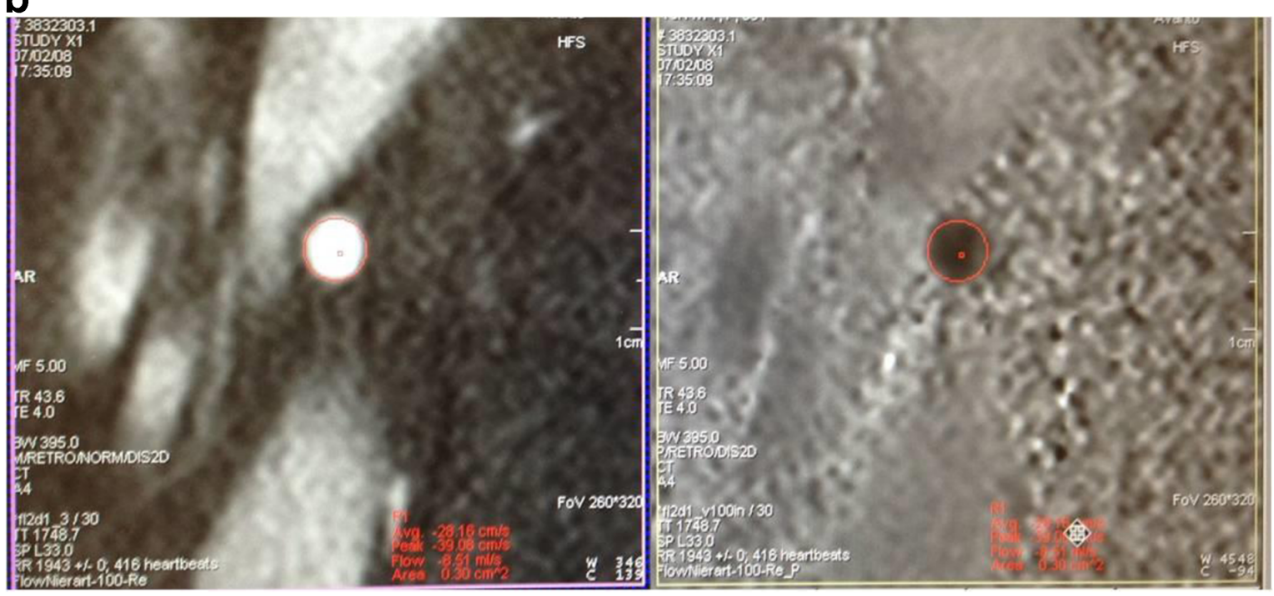

C

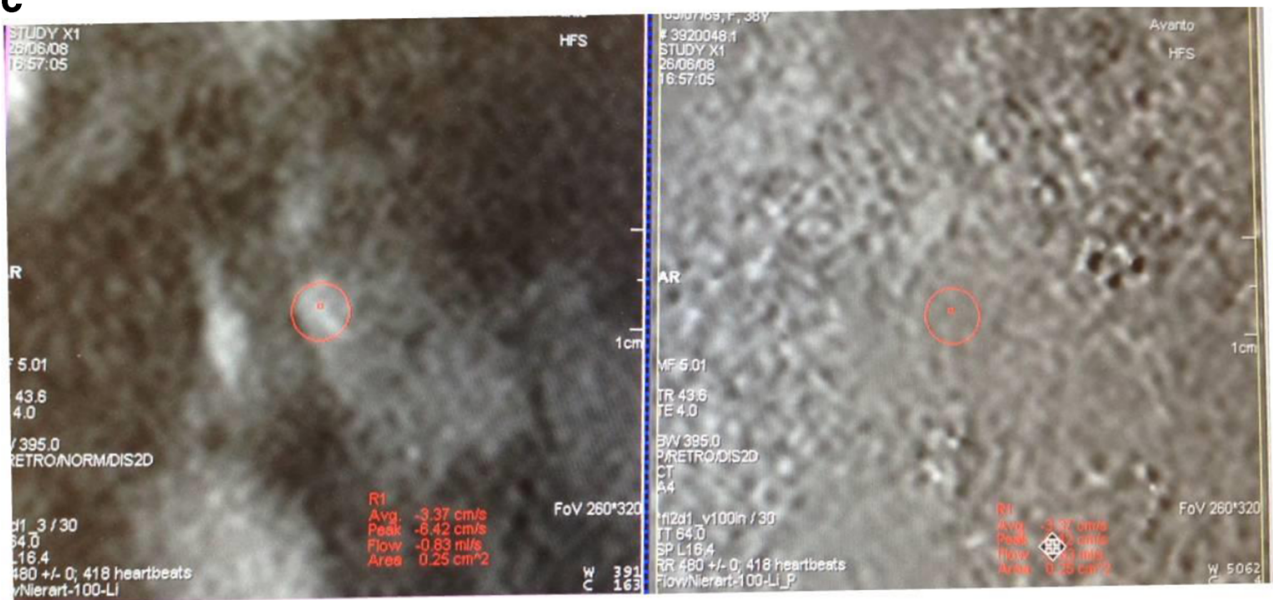

high eGFR. Fourth, $\mathrm{RBF}_{\mathrm{MRI}}$ measurement in patients with lower eGFRs resulted less often in interpretable images. MRI images were judged technically unsatisfactory in $34 \%$ of patients with an eGFR $\leq 70$ vs. in $16 \%$ of patients with an eGFR $>70 \mathrm{ml} / \mathrm{min}^{*} 1.73 \mathrm{~m}^{2}$. Fifth, in subjects with a lower eGFR, only a small fraction of variability in $\mathrm{RBF}_{\mathrm{MRI}}$ was explained by disease characteristics. We speculate that due to the lower RBF in smaller renal arteries the phase-contrast measurement may give a (too) weak signal, causing the lower success rate. Two methods could be used to overcome this problem. The spatial resolution could be increased or the VENC could be adjusted. However, for this study, we used the protocol of the CRISP consortium $[6,7,19]$ with predetermined variables (FOV $32 \mathrm{~cm}$, slide thickness $5 \mathrm{~mm}$, VENC of $100 \mathrm{~cm} / \mathrm{s}$ ) as to maintain the protocol and to compare results without bias. In addition, renal arteries with larger 
Table 2 Associations of renal blood flow $\left(\mathrm{ml} / \mathrm{min}^{*} 1.73 \mathrm{~m}^{2}\right)$ measured by MRI or hippuran with characteristics indicative for disease severity in ADPK $\mathrm{D}(\mathrm{n}=91)$

\begin{tabular}{|c|c|c|c|c|c|c|c|c|}
\hline & \multicolumn{4}{|c|}{ Univariate analyses } & \multicolumn{4}{|c|}{ Multivariate analyses } \\
\hline & \multicolumn{2}{|l|}{ MRI } & \multicolumn{2}{|c|}{ Hippuran } & \multicolumn{2}{|l|}{ MRI } & \multicolumn{2}{|c|}{ Hippuran } \\
\hline & $\mathrm{r}$ & $p$ & $\mathrm{r}$ & $p$ & Std $\beta$ & $p$ & $\operatorname{Std} \beta$ & $p$ \\
\hline Adjusted $\mathrm{R}^{2}$ & & & & & $0.61^{*}$ & & $0.77 *$ & \\
\hline Age (years) & -0.56 & $<0.001$ & -0.58 & $<0.001$ & & & -0.10 & 0.17 \\
\hline Female vs male sex ( 1 or 0$)$ & 0.096 & 0.37 & -0.06 & 0.58 & & & -0.11 & 0.09 \\
\hline Weight (kg) & -0.21 & 0.05 & -0.13 & 0.23 & & & -0.09 & 0.15 \\
\hline Systolic blood pressure (mmHg) & -0.07 & 0.54 & 0.12 & 0.25 & -0.09 & 0.16 & 0.07 & 0.21 \\
\hline Antihypertensive drugs (Yes=1) & -0.15 & 0.16 & -0.18 & 0.08 & 0.13 & 0.08 & 0.11 & 0.05 \\
\hline Plasma osmolality (mOsm/kg) & -0.30 & 0.004 & -0.14 & 0.18 & & & 0.09 & 0.14 \\
\hline Log Copeptin (pmol/l) & -0.24 & 0.02 & -0.16 & 0.13 & -0.07 & 0.31 & & \\
\hline eGFR (ml/min* $\left.1.73 \mathrm{~m}^{2}\right)$ & 0.77 & $<0.001$ & 0.86 & $<0.001$ & 0.82 & $<0.001$ & 0.88 & $<0.001$ \\
\hline Ln TKV/h (ml/m) & -0.22 & 0.04 & -0.23 & 0.03 & & & & \\
\hline 24h urine volume $(\mathrm{mL})$ & -0.17 & 0.10 & -0.10 & 0.33 & & & 0.07 & 0.25 \\
\hline Urinary sodium excretion (mmol/24h) & 0.23 & 0.03 & 0.30 & 0.003 & & & & \\
\hline Ln Urinary albumin excretion (mg/24h) & 0.04 & 0.73 & -0.02 & 0.86 & 0.14 & 0.04 & & \\
\hline
\end{tabular}

$\mathrm{TKV} / \mathrm{h}$ and UAE were natural logarithm transformed and copeptin was log transformed. Abbreviations: eGFR, estimated glomerular filtration rate; $\mathrm{TKV} / \mathrm{h}$, total kidney volume/height. * Model discrimination assessed by bootstrapping (2000 times), $p$-value $<0.001$

Table 3 Multivariate associations of renal blood flow $\left(\mathrm{ml} / \mathrm{min}^{*} 1.73 \mathrm{~m}^{2}\right)$ measured by MRI or hippuran with characteristics indicative for disease severity in patients stratified for kidney function

\begin{tabular}{|c|c|c|c|c|}
\hline \multirow{2}{*}{$\begin{array}{l}\mathrm{eGFR}>70 \mathrm{ml} / \mathrm{min}^{*} 1.73 \mathrm{~m}^{2} \\
\mathrm{~N}=57\end{array}$} & \multicolumn{2}{|l|}{ MRI } & \multicolumn{2}{|c|}{ Hippuran } \\
\hline & $\operatorname{Std} \beta$ & $p$ & Std $\beta$ & $p$ \\
\hline Adjusted $\mathrm{R}^{2}$ & 0.36 & & 0.51 & \\
\hline Age (years) & & & -0.26 & 0.07 \\
\hline Female vs male sex ( 1 or 0$)$ & & & -0.36 & 0.005 \\
\hline Weight $(\mathrm{kg})$ & -0.13 & 0.23 & -0.20 & 0.08 \\
\hline Antihypertensive drugs $(Y e s=1)$ & 0.19 & 0.01 & 0.17 & 0.11 \\
\hline Plasma osmolality (mOsm/kg) & & & 0.14 & 0.20 \\
\hline eGFR $\left(\mathrm{ml} / \mathrm{min}^{*} 1.73 \mathrm{~m}^{2}\right)$ & 0.66 & $<0.001$ & 0.60 & $<0.001$ \\
\hline $24 \mathrm{~h}$ Urine volume $(\mathrm{mL})$ & 0.12 & 0.30 & 0.28 & 0.02 \\
\hline Ln (Urinary albumin excretion $\mathrm{mg} / 24 \mathrm{~h}$ ) & 0.24 & 0.03 & 0.16 & 0.13 \\
\hline $\mathrm{eGFR} \leq 70 \mathrm{ml} / \mathrm{min}^{*} 1.73 \mathrm{~m}^{2}$ & MRI & & Hippura & \\
\hline $\mathrm{N}=34$ & Std $\beta$ & $p$ & Std $\beta$ & $p$ \\
\hline Adjusted $\mathrm{R}^{2}$ & $0.17^{*}$ & & $0.72 *$ & \\
\hline Female vs male sex ( 1 or 0$)$ & -0.30 & 0.15 & & \\
\hline Weight $(\mathrm{kg})$ & -0.21 & 0.24 & & \\
\hline Plasma osmolality (mOsm/kg) & -0.23 & 0.20 & 0.10 & 0.31 \\
\hline Log (Copeptin pmol/l) & -0.19 & 0.30 & -0.25 & 0.03 \\
\hline eGFR $\left(\mathrm{ml} / \mathrm{min}^{*} 1.73 \mathrm{~m}^{2}\right)$ & 0.38 & 0.03 & 0.89 & $<0.001$ \\
\hline $24 \mathrm{~h}$ Urine volume $(\mathrm{mL})$ & -0.22 & 0.20 & -0.23 & 0.02 \\
\hline Ln (Urinary albumin excretion $\mathrm{mg} / 24 \mathrm{~h}$ ) & & & 0.17 & 0.14 \\
\hline
\end{tabular}

* Model discrimination assessed by bootstrapping (2000 times), $p$-value $<0.001$. 
diameters allow better phase-contrast positioning and easier measurement of the flow. Notwithstanding these observations, we think that this does not necessarily disqualify the use of $\mathrm{RBF}_{\mathrm{MRI}}$ measurement for ADPKD staging and risk classification. The clinical need for risk classification in this patient group is especially present early in the disease, when GFR remains within the normal limits for an extended period of time [1]. At a later stage of the disease, when kidney function starts to decline, eGFR in association with age can be used as a parameter to assess disease severity and prognosis. It should be stated that in patients with an eGFR $>70 \mathrm{ml} / \mathrm{min}^{*} 1.73 \mathrm{~m}^{2}$, gadolinium-enhanced techniques can also be used to measure RBF, because in this patient subgroup there is a low risk of toxicity. Lastly, there are other unenhanced techniques to measure flow, such as arterial spin labelling [25], but this technique is especially useful to measure regional variations in renal perfusion, and investigating this technique was beyond the scope of the present study. Of note, a pilot study showed no difference for RBF values between arterial spin labelling and $\mathrm{RBF}_{\mathrm{MRI}}$ measurement [26].

Limitations of our study are, first, that we studied renal hemodynamical parameters in a cross-sectional setting. Therefore, we were not able to investigate associations with disease progression. Second, this study assessed reliability and accuracy of $\mathrm{RBF}_{\mathrm{MRI}}$ using a phantom simulating the clinical situation. However, it lacks a gold standard method for RBF measurement in vivo. Such a method would necessitate a flow probe directly introduced in the renal arteries, which, because of its invasive character, is not feasible. We, therefore, assessed reliability of $\mathrm{RBF}_{\mathrm{MRI}}$ in an indirect way, by studying the degree of variability of $\mathrm{RBF}_{\mathrm{MRI}}$ that was explained by accepted characteristics of ADPKD severity, and by comparing this with the degree of variability of $\mathrm{RBF}_{\mathrm{Hip}}$ explained by the same disease characteristics. Strengths of our study were that we measured $\mathrm{RBF}_{\mathrm{MRI}}$ and ERPF by gold-standard techniques on the same day. These measurements were performed in a relatively large, well-phenotyped cohort of ADPKD patients with relatively preserved as well as impaired kidney function. Furthermore, our method avoids use of gadolinium, which is important because of the potential side effects of this contrast agent in patients with kidney dysfunction [27].

In conclusion, our study shows that in ADPKD patients, RBF can be measured by MRI accurately and reliably without using gadolinium contrast, but that assessment of RBF by PC-MRI may be less feasible in patients with impaired kidney function.

\footnotetext{
Acknowledgements The scientific guarantor of this publication is Ron T. Gansevoort. The authors of this manuscript declare relationships with the following companies: Otzuka. The authors of this manuscript declare no relationships with any companies whose products or services may be related to the subject matter of the article. The authors state that this work has not received any funding. One of the authors has significant statistical expertise. Institutional review board approval was obtained. Written informed consent was obtained from all subjects (patients) in this study.
}

Some study subjects or cohorts have been previously reported in

- Clin J Am Soc Nephrol. 2010 Jun;5(6):1091-8. doi: 10.2215/CJN. 00360110

-Am J Kidney Dis. 2013 Sep;62(3):531-40. doi: 10.1053/j.ajkd.2013. 03.030.

Methodology: retrospective, cross sectional study, performed at one institution.

Open Access This article is distributed under the terms of the Creative Commons Attribution-NonCommercial 4.0 International License (http://creativecommons.org/licenses/by-nc/4.0/), which permits any noncommercial use, distribution, and reproduction in any medium, provided you give appropriate credit to the original author(s) and the source, provide a link to the Creative Commons license, and indicate if changes were made.

\section{References}

1. Grantham JJ (2008) Clinical practice. autosomal dominant polycystic kidney disease. N Engl J Med 359:1477-85

2. Torres VE, Chapman AB, Devuyst O et al (2012) Tolvaptan in patients with autosomal dominant polycystic kidney disease. $\mathrm{N}$ Engl J Med 367:2407-18

3. Caroli A, Perico N, Perna A et al (2013) Effect of long-acting somatostatin analogue on kidney and cyst growth in autosomal dominant polycystic kidney disease (ALADIN): A randomised, placebo-controlled, multicentre trial. Lancet 382:1485-95

4. Grantham JJ, Mulamalla S, Swenson-Fields KI (2011) Why kidneys fail in autosomal dominant polycystic kidney disease. Nat Rev Nephrol 7:556-66

5. Grantham JJ, Torres VE, Chapman AB et al (2006) Volume progression in polycystic kidney disease. N Engl J Med 354:2122-30

6. King BF, Torres VE, Brummer ME et al (2003) Magnetic resonance measurements of renal blood flow as a marker of disease severity in autosomal-dominant polycystic kidney disease. Kidney Int 64 : 2214-21

7. Torres VE, King BF, Chapman AB et al (2007) Magnetic resonance measurements of renal blood flow and disease progression in autosomal dominant polycystic kidney disease. Clin J Am Soc Nephrol 2:112-20

8. Ergun I, Keven K, Uruc I et al (2006) The safety of gadolinium in patients with stage 3 and 4 renal failure. Nephrol Dial Transplant 21:697-700

9. Bernstein EJ, Schmidt-Lauber C, Kay J (2012) Nephrogenic systemic fibrosis: A systemic fibrosing disease resulting from gadolinium exposure. Best Pract Res Clin Rheumatol 26:489-503

10. Penfield JG, Reilly RF Jr (2007) What nephrologists need to know about gadolinium. Nat Clin Pract Nephrol 3:654-68

11. Donker AJ, van der Hem GK, Sluiter WJ, Beekhuis H (1977) A radioisotope method for simultaneous determination of the glomerular filtration rate and the effective renal plasma flow. Neth J Med 20:97-103

12. Battilana C, Zhang HP, Olshen RA, Wexler L, Myers BD (1991) PAH extraction and estimation of plasma flow in diseased human kidneys. Am J Physiol 261:F726-33

13. Pei Y, Obaji J, Dupuis A et al (2009) Unified criteria for ultrasonographic diagnosis of ADPKD. J Am Soc Nephrol 20:205-12

14. Levey AS, Stevens LA, Schmid CH et al (2009) A new equation to estimate glomerular filtration rate. Ann Intern Med 150:604-12 
15. Levey AS, Greene T, Kusek J, Beck GJ A simplified equation to predict glomerular filtration rate for serum creatinine [abstract]. 2000:11:115A.

16. Bae KT, Commean PK, Lee J (2000) Volumetric measurement of renal cysts and parenchyma using MRI: Phantoms and patients with polycystic kidney disease. J Comput Assist Tomogr 24:614-9

17. de Haan MW, Kouwenhoven M, Kessels AG, van Engelshoven JM (2000) Renal artery blood flow: Quantification with breath-hold or respiratory triggered phase-contrast MR imaging. Eur Radiol 10: 1133-7

18. Bax L, Bakker CJ, Klein WM, Blanken N, Beutler JJ, Mali WP (2005) Renal blood flow measurements with use of phase-contrast magnetic resonance imaging: Normal values and reproducibility. $\mathrm{J}$ Vasc Interv Radiol 16:807-14

19. Dambreville S, Chapman AB, Torres VE et al (2010) Renal arterial blood flow measurement by breath-held MRI: Accuracy in phantom scans and reproducibility in healthy subjects. Magn Reson Med 63:940-50

20. Apperloo AJ, de Zeeuw D, Donker AJ, de Jong PE (1996) Precision of glomerular filtration rate determinations for long-term slope calculations is improved by simultaneous infusion of 125I-iothalamate and 131I-hippuran. J Am Soc Nephrol 7:567-72

21. Cameron AC, Windmeijer FAG (1996) R-squared measures for count data regression models with applications to health-care utilization. J Bus Econ Stat 14:209-20
22. Sommer G, Corrigan G, Fredrickson J et al (1998) Renal blood flow: Measurement in vivo with rapid spiral MR imaging. Radiology 208:729-34

23. Wolf RL, King BF, Torres VE, Wilson DM, Ehman RL (1993) Measurement of normal renal artery blood flow: Cine phasecontrast MR imaging vs clearance of p-aminohippurate. AJR Am J Roentgenol 161:995-1002

24. Cutajar M, Hilton R, Olsburgh J et al (2015) Renal blood flow using arterial spin labelling MRI and calculated filtration fraction in healthy adult kidney donors pre-nephrectomy and post-nephrectomy. Eur Radiol. doi:10.1007/s00330-0153594-6

25. Ferre JC, Bannier E, Raoult H, Mineur G, Carsin-Nicol B, Gauvrit JY (2013) Arterial spin labeling (ASL) perfusion: Techniques and clinical use. Diagn Interv Imaging 94:1211-23

26. Cutajar M, Thomas DL, Hales PW, Banks T, Clark CA, Gordon I (2014) Comparison of ASL and DCE MRI for the non-invasive measurement of renal blood flow: Quantification and reproducibility. Eur Radiol 24:1300-8

27. Anonymous (12/23/2010) FDA drug safety communication: New warnings for using gadolinium-based contrast agents in patients with kidney dysfunction. :http://www.fda.gov/Drugs/DrugSafety/ ucm223966.htm 